\title{
Quantum fidelity and quantum phase transitions in matrix product states
}

\author{
Marco Cozzini, ${ }^{1,2}$ Radu Ionicioiu, ${ }^{2}$ and Paolo Zanardi ${ }^{2,3}$ \\ ${ }^{I}$ Dipartimento di Fisica, Politecnico di Torino, Corso Duca degli Abruzzi 24, I-10129 Torino, Italy \\ ${ }^{2}$ Quantum Information Group, Institute for Scientific Interchange (ISI), Viale Settimio Severo 65, I-10133 Torino, Italy \\ ${ }^{3}$ Department of Physics and Astronomy, University of Southern California, Los Angeles, CA 90089-0484, USA
}

\begin{abstract}
Matrix product states, a key ingredient of numerical algorithms widely employed in the simulation of quantum spin chains, provide an intriguing tool for quantum phase transition engineering. At critical values of the control parameters on which their constituent matrices depend, singularities in the expectation values of certain observables can appear, in spite of the analyticity of the ground state energy. For this class of generalized quantum phase transitions we test the validity of the recently introduced fidelity approach, where the overlap modulus of ground states corresponding to slightly different parameters is considered. We discuss several examples, successfully identifying all the present transitions. We also study the finite size scaling of fidelity derivatives, pointing out its relevance in extracting critical exponents.
\end{abstract}

PACS numbers: 05.30.-d,64.60.-i

\section{INTRODUCTION}

The study of many-body quantum systems is at the same time a fascinating and challenging field of research. This is due to the richness of inherently complex phenomena arising in the presence of a large number of interacting particles, among which quantum phase transitions (QPTs) occupy a distinguished position. These transitions take place at zero temperature and are driven by an external parameter, as for example the magnetic field in superconductors.

Recently it has been shown that the quantum fidelity - the overlap modulus - of two finite size ground states corresponding to neighboring control parameters is a good indicator of QPTs. Indeed, the fidelity typically drops abruptly at critical points, as a consequence of the dramatic state transformation involved in a transition. This approach has been tested in various contexts where the ground state can be calculated exactly, either analytically or numerically. These include Dicke model, one-dimensional $X Y$ model in a transverse field, and general quadratic fermionic Hamiltonians ${ }^{1,2}$.

In the present article we further pursue this approach by analyzing QPTs described by matrix product states (MPSs) ${ }^{3}$. These states are at the basis of efficient numerical methods used in the analysis of spin chain systems, as the density matrix renormalization group (DMRG) algorithm. When considered dependent on a control parameter $g$ they can give rise to generalized QPTs, i.e., transitions where some observable quantity presents a non-analytic behavior in spite of the regularity of the ground state energy ${ }^{4}$. We show that the quantum fidelity of two neighboring (in terms of $g$ ) MPSs is an effective tool not only in detecting the critical point $g_{c}$ but also in giving the correct scaling at $g_{c}$. The success of the fidelity approach in analyzing MPS-QPTs further proves the generality of the procedure, which, as stressed in Refs. 1,2, does not require any a priori understanding of the structure (order parameter, correlation functions, topology, etc.) of the considered system. It is also worth pointing out that this method seems to have some advantage with respect to other quantum information based techniques. For example, quantum phase transitions in MPSs do not give rise to the logarithmic divergence of the entropy of block entanglement observed in other systems ${ }^{5}$, thereby ruling out this quantity as a reliable transition indicator. On the other hand, other entanglement measures can be used, e.g., single site entanglement and concurrence, whose derivatives often provide interesting information about criticality ${ }^{6}$. We discuss the results obtained by these methods in one of the simple examples we analyze below. As it will be shown, also these latter measures turn to be less effective than the fidelity approach.

In the following we will first present a derivation of the overlap formula for general MPSs (Sec. III), discussing some general features of these systems, and then study in detail the MPS examples introduced in Ref.4 (Sec.III). As in Refs. 1.2, the analysis will be carried out also in terms of fidelity derivatives, which are the most suitable tool to observe finite size scaling properties. In particular, we will explicitly show how to extract the critical exponent of the correlation length from these quantities (Subsec. IIIA), thus demonstrating the independence and the completeness of our approach. Finally, we will report on the entanglement analysis in Subsec. IIIB.

\section{FIDELITY FOR MATRIX PRODUCT STATES}

Suppose we have a closed spin chain with $N$ sites. Let $d$ be the dimension of the Hilbert space at each site. The matrix product states are defined as ${ }^{4}$

$$
|g\rangle:=|\psi(g)\rangle=\frac{1}{\sqrt{\mathcal{N}}} \sum_{i_{1}, \ldots, i_{N}=0}^{d-1} \operatorname{Tr}\left(A_{i_{1}} \ldots A_{i_{N}}\right)\left|i_{1} \ldots i_{N}\right\rangle
$$

where the $A_{j}$ 's, with $j=0, \ldots, d-1$, are $D \times D$ matrices, $D$ is the dimension of the bonds in the so-called valence bond picture, and $\mathcal{N}:=\sum_{i_{1}, \ldots, i_{N}=0}^{d-1}\left|\operatorname{Tr}\left(A_{i_{1}} \ldots A_{i_{N}}\right)\right|^{2}$ a normalization factor. Since our goal is to explore quantum phase transitions in such states, we assume that the matrices $A_{j}=A_{j}(g)$ depend on one or more parameters, generically denoted by $g$. The overlap of two MPSs corresponding to dif- 
ferent $g$ 's is given by

$$
\begin{aligned}
& \left\langle g_{1} \mid g_{2}\right\rangle=\left[\mathcal{N}\left(g_{1}\right) \mathcal{N}\left(g_{2}\right)\right]^{-1 / 2} \\
& \quad \times \sum_{i \in \mathbb{Z}_{d}^{N}} \operatorname{Tr}\left[A_{i_{1}}^{*}\left(g_{1}\right) \ldots A_{i_{N}}^{*}\left(g_{1}\right)\right] \operatorname{Tr}\left[A_{i_{1}}\left(g_{2}\right) \ldots A_{i_{N}}\left(g_{2}\right)\right]
\end{aligned}
$$

where $\boldsymbol{i}=\left(i_{1}, \ldots, i_{N}\right)$. In order to simplify the formulæ, we will work with the unnormalized overlap

$$
F\left(g_{1}, g_{2}\right):=\sqrt{\mathcal{N}\left(g_{1}\right) \mathcal{N}\left(g_{2}\right)}\left\langle g_{1} \mid g_{2}\right\rangle
$$

and we obviously have $\mathcal{N}\left(g_{a}\right)=F\left(g_{a}, g_{a}\right), a=1,2$. Using the identity $\operatorname{Tr}(A) \operatorname{Tr}(B)=\operatorname{Tr}(A \otimes B)$, the overlap can be computed exactly for MPSs ${ }^{7}$ and we obtain

$$
F\left(g_{1}, g_{2}\right)=\operatorname{Tr}\left[E^{N}\left(g_{1}, g_{2}\right)\right]=\sum_{k=1}^{D^{2}} \lambda_{k}^{N}\left(g_{1}, g_{2}\right),
$$

where $\lambda_{k}$ are the eigenvalues of the $D^{2} \times D^{2}$ matrix

$$
E\left(g_{1}, g_{2}\right):=\sum_{i=0}^{d-1} A_{i}^{*}\left(g_{1}\right) \otimes A_{i}\left(g_{2}\right) .
$$

The latter is the generalization of the transfer operator $E_{\mathbb{1}}(g):=E(g, g)$, which we assume to be diagonalizable with eigenvalues $\lambda_{k}(g):=\lambda_{k}(g, g)$. Also, since $A \otimes B$ and $B \otimes A$ are isospectral, one has $F\left(g_{1}, g_{2}\right)=F\left(g_{2}, g_{1}\right)^{*}$.

The main object of our study will be the fidelity of two neighboring states

$$
\mathcal{F}(g ; \delta):=|\langle g-\delta \mid g+\delta\rangle|=\frac{|F(g-\delta, g+\delta)|}{\sqrt{\mathcal{N}(g-\delta) \mathcal{N}(g+\delta)}}
$$

for a small variation $\delta$ in the parameter space spanned by $g$. Defined this way, the fidelity depends not only on the parameter $g$ driving the QPT, but also on its variation $\delta$. Expanding the fidelity in $\delta$ we obtain $\mathcal{F}(g ; \delta) \simeq 1+\left.\partial_{\delta}^{2} \mathcal{F}(g ; \delta)\right|_{\delta=0} \delta^{2} / 2$ (since $\mathcal{F}(g ; \delta)$ reaches its maximum for $\delta=0$ the first derivative vanishes). Thus the rate of change of the fidelity close to a critical point is given by the second derivative of the fidelity ${ }^{1,2}$

$$
S(g):=\left.\partial_{\delta}^{2} \mathcal{F}(g ; \delta)\right|_{\delta=0}
$$

and this is the relevant quantity we will study to determine the scaling at the critical point $g=g_{c}$. Note that $S(g)$ is connected to the ground state variation $\left|\psi^{\prime}(g)\right\rangle=\partial_{g}|\psi(g)\rangle$. For example, for real states one has the simple second order expansion $\mathcal{F}(g ; \delta) \simeq 1-2 \delta^{2}\left\|\psi^{\prime}(g)\right\|^{2}$. The general expression of the second derivative has the form 12

$$
S(g)=-\left.4 \partial_{g_{1}} \partial_{g_{2}} \ln F\left(g_{1}, g_{2}\right)\right|_{g_{1}=g_{2}=g} .
$$

Indeed, from $\mathcal{F}(g ; 0)=1$ and $\left.\partial_{\delta} \mathcal{F}(g ; \delta)\right|_{\delta=0}=0$ one has $\left.\partial_{\delta}^{2} \ln \mathcal{F}(g ; \delta)\right|_{\delta=0}=\left.\partial_{\delta}^{2} \mathcal{F}(g ; \delta)\right|_{\delta=0}=S(g)$ and then

$$
\begin{aligned}
S(g) & =\left.\frac{1}{2} \partial_{\delta}^{2} \ln \frac{F(g-\delta, g+\delta) F(g+\delta, g-\delta)}{F(g-\delta, g-\delta) F(g+\delta, g+\delta)}\right|_{\delta=0}= \\
& =\left.\left(\partial_{\delta}^{2}-\partial_{g}^{2}\right) \ln F(g-\delta, g+\delta)\right|_{\delta=0}
\end{aligned}
$$

from which Eq. (8) follows immediately.

The general behavior of the fidelity for matrix product states in the thermodynamic limit (TDL) can be inferred from Eq. (4). Without loss of generality we can assume the eigenvalues $\lambda_{k}$ of the generalized transfer operator $E$ to be ordered $\left|\lambda_{1}\right| \geq\left|\lambda_{2}\right| \geq \ldots \geq\left|\lambda_{D^{2}}\right|$. Then $F\left(g_{1}, g_{2}\right)=$ $\sum_{k=1}^{D^{2}} \lambda_{k}^{N}\left(g_{1}, g_{2}\right)=\lambda_{1}^{N}\left[1+\sum_{k=2}^{D^{2}}\left(\lambda_{k} / \lambda_{1}\right)^{N}\right]$ and, provided $\left|\lambda_{1}\right|>\left|\lambda_{k}\right|$ for $k=2, \ldots, D^{2}$, in the thermodynamic limit $N \rightarrow \infty$ one finds $F\left(g_{1}, g_{2}\right) \sim \lambda_{1}^{N}$. Since the same holds for the normalization factors $\mathcal{N}(g)=F(g, g)$, whenever the maximum eigenvalues $\lambda_{1}$ are non-degenerate, the large $N$ behavior of the fidelity must be of the form $\mathcal{F}(g ; \delta) \sim \alpha^{N}$, with $0 \leq \alpha \leq 1$ by construction. Typically, $\alpha \neq 1$ and the fidelity decays exponentially in the TDL $\stackrel{7}{ }$. If $E_{\mathbb{1}}(g)$ exhibits a level crossing in the largest eigenvalue for a critical coupling $g_{c}$, the degeneracy $\left|\lambda_{1}\left(g_{c}\right)\right|=\left|\lambda_{2}\left(g_{c}\right)\right|$ gives rise to a discontinuity of some expectation values in the TDL (called a generalized QPT in Ref.4). In this case the previous discussion has to be modified in order to include all the degenerate eigenvalues. In general, the vanishing of the fidelity is typically strongly enhanced at critical points.

A similar analysis can be done for the second derivative $S(g)$ of the fidelity. When a single eigenvalue $\lambda_{1}$ dominates, in the TDL one can replace $F$ with $\lambda_{1}^{N}$ in Eq. (8), thereby recovering the expected ${ }^{2}$ linear scaling $\propto N$ :

$$
S(g) \sim-\left.4 N \partial_{g_{1}} \partial_{g_{2}} \ln \lambda_{1}\left(g_{1}, g_{2}\right)\right|_{g_{1}=g_{2}=g} .
$$

In contrast, at the critical point two or more eigenvalues of $E\left(g_{1}, g_{2}\right)$ are equal in modulus. For example, at $g=g_{c}$ one can have $\left|\lambda_{1}\left(g_{c}\right)\right|=\left|\lambda_{2}\left(g_{c}\right)\right|>\left|\lambda_{k}\left(g_{c}\right)\right|$ for $k>2$. Then in the TDL

$$
\begin{aligned}
S\left(g_{c}\right) \sim & \lim _{g \rightarrow g_{c}}-\left.4 \partial_{g_{1}} \partial_{g_{2}} \ln \left(\lambda_{1}^{N}+\lambda_{2}^{N}\right)\right|_{g_{1}=g_{2}=g}= \\
= & \lim _{g \rightarrow g_{c}}\left\{-N^{2} \frac{4 \lambda_{1}^{N} \lambda_{2}^{N}}{\left(\lambda_{1}^{N}+\lambda_{2}^{N}\right)^{2}} \partial_{g_{1}} \ln \frac{\lambda_{1}}{\lambda_{2}} \partial_{g_{2}} \ln \frac{\lambda_{1}}{\lambda_{2}}\right. \\
& \left.-4 N \sum_{k=1}^{2} \frac{\lambda_{k}^{N}}{\lambda_{1}^{N}+\lambda_{2}^{N}} \partial_{g_{1}} \partial_{g_{2}} \ln \lambda_{k}\right\}\left.\right|_{g_{1}=g_{2}=g},
\end{aligned}
$$

where $\lambda_{1,2}=\lambda_{1,2}\left(g_{1}, g_{2}\right)$. Hence, at the transition one typically recovers the divergence $S\left(g_{c}\right) \propto N^{2}$ already observed in Ref. 2. We also note that, by defining $\theta:=\ln \left(\lambda_{1} / \lambda_{2}\right)$, the term in the second line of Eq. (11) can be rewritten in the compact form $\left[N\left|\partial_{g_{1}} \theta\right| / \cosh (N \theta / 2)\right]^{2}$. This follows from the equality $\partial_{g_{1}} \ln \left(\lambda_{1} / \lambda_{2}\right) \partial_{g_{2}} \ln \left(\lambda_{1} / \lambda_{2}\right)=\left|\partial_{g_{1}} \ln \left(\lambda_{1} / \lambda_{2}\right)\right|^{2}$, due to the relation $\lambda_{1,2}\left(g_{2}, g_{1}\right)=\lambda_{1,2}^{*}\left(g_{1}, g_{2}\right)$. Then, it is clear that this term vanishes for $N \rightarrow \infty$ unless $\Re \theta=0$, i.e., $\left|\lambda_{1}\right|=\left|\lambda_{2}\right|$. In the latter case, instead, assuming $\partial_{g_{1}} \theta$ to be finite, the scaling $\propto N^{2}$ is evident. Finally, we point out that the limit $g \rightarrow g_{c}$ in Eq. (11) is necessary to deal with possible singularities in the derivatives of the $\lambda_{k}$ 's at the transition (see the examples below).

\section{EXAMPLES}

In the following we are going to study the explicit examples of MPSs described in Ref. 4. Without loss of generality we 


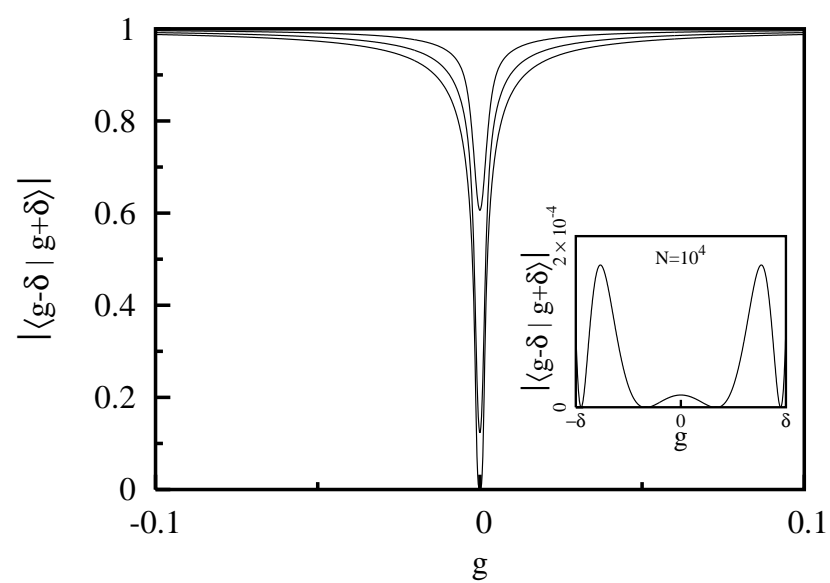

FIG. 1: Fidelity $\mathcal{F}(g ; \delta)=|\langle g-\delta \mid g+\delta\rangle|$ as a function of $g$ for Example 1 with $\delta=10^{-3}$ and $N=1000,2000,3000$. Inset: close to the critical point, the fidelity oscillates for states belonging to different phases $\left(\delta=10^{-3}, N=10^{4}\right)$.

assume $g_{1}=g-\delta<g_{2}=g+\delta$.

\section{A. Fidelity and second order MPS-QPTs}

Example 1. Take $D=2, d=3$ and $\left\{A_{j}\right\}=$ $\left\{-Z, \sigma_{-}, g \sigma_{+}\right\}$; in the following we will use the notation $X, Z$ for the Pauli matrices, $\sigma_{ \pm}$being the corresponding raising and lowering operators. This one-parameter family of MPSs contains the ground state of spin-1 AKLT model for $g= \pm 2$ and has a critical point at $g_{c}=0$ (see Ref. 4). The model is also significant because it has non-local string order. The parent Hamiltonian with two-body interaction is given by

$$
\begin{aligned}
H= & \sum_{i}\left[\left(2+g^{2}\right) \boldsymbol{S}_{i} \cdot \boldsymbol{S}_{i+1}+2\left(\boldsymbol{S}_{i} \cdot \boldsymbol{S}_{i+1}\right)^{2}+\right. \\
& +2\left(4-g^{2}\right)\left(S_{i}^{z}\right)^{2}+(g+2)^{2}\left(S_{i}^{z} S_{i+1}^{z}\right)^{2}+ \\
& \left.+g(g+2)\left\{S_{i}^{z} S_{i+1}^{z}, \boldsymbol{S}_{i} \cdot \boldsymbol{S}_{i+1}\right\}\right],
\end{aligned}
$$

where $\boldsymbol{S}_{i}$ is the spin operator acting on the $i$-th site. The eigenvalues of the transfer operator $E=Z \otimes Z+\sigma_{-} \otimes \sigma_{-}+$ $g_{1} g_{2} \sigma_{+} \otimes \sigma_{+}$are $\left(-1,-1,1 \pm \sqrt{g_{1} g_{2}}\right)$ and Eq. (4) yields

$$
\begin{gathered}
F\left(g_{1}, g_{2}\right)=\left(1+\sqrt{g_{1} g_{2}}\right)^{N}+\left(1-\sqrt{g_{1} g_{2}}\right)^{N}+2(-1)^{N} \\
\text { and } \mathcal{N}(g)=F(g, g)=(1+g)^{N}+(1-g)^{N}+2(-1)^{N} .
\end{gathered}
$$

Let us analyze the behavior of the fidelity $\mathcal{F}(g ; \delta)$ defined in Eq. (6). Since $\mathcal{F}(g ; \delta)$ is evidently symmetric in both $g$ and $\delta$, we can safely assume $g \geq 0, \delta>0$. Three cases can then be distinguished, according to the behavior of the eigenvalues of the transfer operators:

(i) $g>\delta$, where $\mathcal{F}(g ; \delta) \sim \alpha^{N}$ for $N \gg 1$, with $\alpha=$ $\left(1+\sqrt{g^{2}-\delta^{2}}\right)^{2} / \sqrt{(1+g-\delta)(1+g+\delta)}<1 ;$

(ii) $g=\delta$, where $\mathcal{F}(\delta ; \delta) \sim a /(1+2 \delta)^{N / 2}$ for $N \gg 1$, with $a=2$ for $N$ even and $a=2 \delta \sqrt{N(N-1)}$ for $N$ odd (this is the case where the largest eigenvalue of $E(g-\delta, g+\delta)$ is

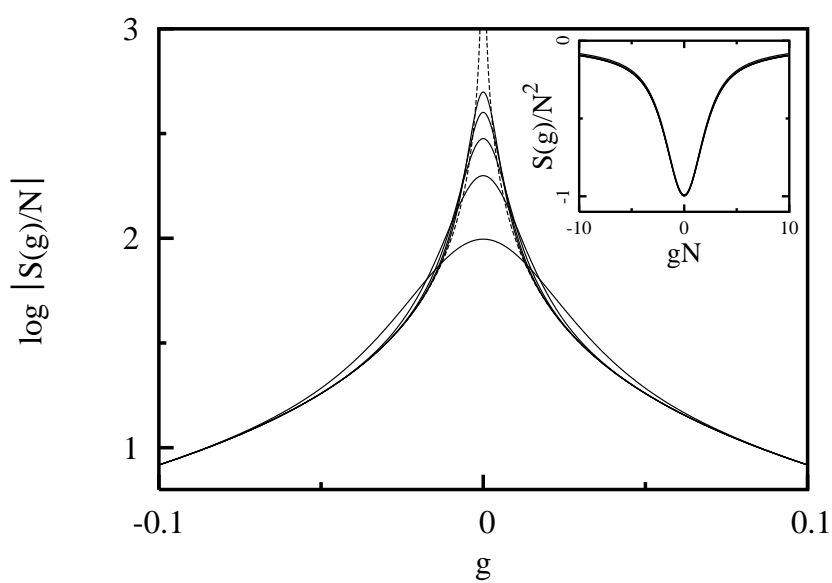

FIG. 2: Finite size scaling for $S(g)$. The second derivative $S(g)$ is plotted for $N=100,200, \ldots, 500$ (logarithm is base 10). The peak at $g=0$ scales with $N$ accordingly to Eq. 16. The dashed line corresponds to the asymptotic behavior given by Eq. (15). The inset shows the data collapsing for the same curves $(N=$ $100,200, \ldots, 500)$ when plotted in rescaled units.

degenerate and the decaying behavior of the fidelity is slightly modified);

(iii) $g<\delta$, where two of the eigenvalues of $E\left(g_{1}, g_{2}\right)$ are complex and, for $N \gg 1$, the fidelity exhibits an oscillatory behavior driven by $|\cos (N \varphi)|, \varphi=$ $\arctan \sqrt{\delta^{2}-g^{2}}$, with a decaying envelope given by $2 \alpha^{N}$, $\alpha=\sqrt{\left(1+\delta^{2}-g^{2}\right) /[(1+\delta-g)(1+\delta+g)]}<1$. Explicitly, at the critical point $g=g_{c}=0$ the overlap becomes

$$
\langle-\delta \mid \delta\rangle=\frac{\left(1+\delta^{2}\right)^{N / 2} \cos \left(N \varphi_{0}\right)+(-1)^{N}}{\left[(1+\delta)^{N}+(1-\delta)^{N}\right] / 2+(-1)^{N}},
$$

where $\varphi_{0}=\arctan \delta$. Then $\mathcal{F}(0 ; \delta)=|\langle-\delta \mid \delta\rangle|$ (note that the overlap can be negative at this point).

The fidelity behavior in the neighborhood of the critical point is shown in Fig. 1 for $\delta=10^{-3}$ and various values of $N$. It is evident that, although for a fixed $\delta$ the fidelity vanishes in the TDL for any $g$, the rate of decrease is much faster at the transition. This fidelity drop provides a useful finite size precursor of the quantum phase transition taking place in the infinite system. In the figure inset we zoom on the oscillatory behavior in the interval $g \in(-\delta, \delta)$, where states on different sides of the transition are considered. Note that oscillations start to be visible only for $N \varphi>\pi$, i.e., $N>N_{\text {osc }}$, $N_{\text {osc }}=\pi / \arctan \sqrt{\delta^{2}-g^{2}}$, which for $g=0$ and $\delta \ll 1$ reduces to $N_{\text {osc }} \sim \pi / \delta$.

The second derivative $S(g)$ of the fidelity can be calculated analytically using formula (8). We find

$$
\begin{aligned}
& S(g \neq 0) \sim-\frac{N}{|g|(1+|g|)^{2}}, \\
& S(g=0)= \begin{cases}-N(N-1) & (N \text { even }) \\
-(N-2)(N-3) / 3 & (N \text { odd })\end{cases}
\end{aligned}
$$


where Eq. (15) corresponds to Eq. 110) and gives the asymptotic behavior for large $N$, while Eq. (16) is exact. As in Ref.2 the critical point is identified by a divergence $\propto N^{2}$ of the second derivative $S(g)$ of the fidelity.

In Fig. 2]we plot log $|S(g)|$ for different values of $N$. In the inset of the figure the curves are plotted in rescaled units, giving rise to data collapsing (lines for different values of $N$ are practically indistinguishable in the inset, merging into a single thick line). This shows that $S(g) / N^{2}$ is basically a function of $g N$ only. This is reminiscent of the usual scaling behavior of diverging observables in second order quantum phase transitions. Indeed, if an observable $P$ of a one-dimensional system diverges algebraically in the TDL for some critical coupling $g_{c}$, i.e., $P_{\infty} \sim c_{\infty}\left|g-g_{c}\right|^{-\rho}$, the finite size scaling Ansatz in the critical region reads $P_{N}=N^{\rho / \nu} Q\left(N\left|g-g_{c}\right|^{\nu}\right)$, where $\nu$ is the critical exponent of the bulk correlation length $\xi_{c}$ and $Q$ is some function ${ }^{8}$. This is due to the fact that at criticality, once properly rescaled by some power of the size of the system, the observable is expected to depend only on the dimensionless ratio $L / \xi_{c}$ between the system size $L \propto N$ and the correlation length. Since the latter diverges at the transition like $\xi_{c} \propto 1 /\left|g-g_{c}\right|^{\nu}$ one immediately finds $L / \xi_{c} \propto N\left|g-g_{c}\right|^{\nu}$. In our case $P_{N}=S / N=N Q(N|g|)$ with $g_{c}=0$, so that we obtain $\rho=\nu=1$. The result $\rho=1$ agrees with Eq. [15, which, for $g \rightarrow 0$, yields $P_{\infty} \sim-|g|^{-1}$. The value of $\nu$ can instead be checked by explicitly calculating the correlation length ${ }^{13}$, where one finds $\nu=1$, confirming the finite size scaling estimate.

The next examples of this subsection correspond to threebody interaction Hamiltonians, again taken from Ref.4. Qualitatively, they all feature the same behavior as Example 1: in the asymptotic limit $|S(g)| / N$ diverges proportionally to $1 /|g|$ at the critical point $g_{c}=0$, while the peak height of $S(g=0)$ scales with $N^{2}$.

Example 2. Consider $D=d=2$ and $A_{0}=(11-Z) / 2+\sigma_{-}$, $A_{0}=(\mathbb{1}+Z) / 2+g \sigma_{+}$. The three-body Hamiltonian is $\mathbb{Z}_{2}$ symmetric and has a critical point at $g_{c}=0$, where the state is a Greenberger-Horne-Zeilinger (GHZ) state, and reads

$H=\sum_{i} 2\left(g^{2}-1\right) Z_{i} Z_{i+1}-(1+g)^{2} X_{i}+(g-1)^{2} Z_{i} X_{i+1} Z_{i+2}$

The generalized transfer operator $E\left(g_{1}, g_{2}\right)$ has eigenvalues $\left(0,0,1 \pm \sqrt{g_{1} g_{2}}\right)$, very similar to Example 1 . The function $F(g-\delta, g+\delta)$ is then still symmetric in $g$ and $\delta$.

Since the largest eigenvalue $\lambda_{1}$ is the same as in Example 1 , the TDL behavior of $\mathcal{F}(g, \delta)$ and $S(g)$ is unchanged. However, the parity dependent term $(-1)^{N}$ is now absent. Then, the asymptotic behavior of $S(g \neq 0)$ is still given by Eq. (15), while the parity dependence of Eq. (16) is lost and one simply has $S(g=0)=-2 N(N-1)$. The latter results follows from Eq. (11), which applies exactly to this case even for finite $N$. Indeed here only two eigenvalues of $E\left(g_{1}, g_{2}\right)$ are different from zero and one has the level crossing $\lambda_{1}(g=0)=\lambda_{2}(g=0)$. Note that, due to the divergence of the double derivative $\partial_{g_{1}} \partial_{g_{2}} \ln \lambda_{1,2}$ calculated in $g_{1}=g_{2}=0$, also the term in the last line of Eq. (11) gives rise to a contribution $\propto N^{2}$, in spite of its apparent linearity in $N$ for $\left|\lambda_{1}\right|=\left|\lambda_{2}\right|$. This explains the necessity of the limit $g \rightarrow g_{c}$ in Eq. 11.

Example 3. Consider $A_{0}=X, A_{1}=\sqrt{g}(11-Z) / 2$. The eigenvalues of $E\left(g_{1}, g_{2}\right)$ are $\left( \pm 1,\left(\sqrt{g_{1} g_{2}} \pm \sqrt{g_{1} g_{2}+4}\right) / 2\right)$. As before, the scaling of the system can be seen from the behavior of $S(g)$ in the thermodynamic limit, driven by $\lambda_{1}\left(g_{1}, g_{2}\right)=\left(\sqrt{g_{1} g_{2}}+\sqrt{g_{1} g_{2}+4}\right) / 2$. We find

$$
\begin{aligned}
& S(g \neq 0) \sim-\frac{4 N}{|g|\left(g^{2}+4\right)^{3 / 2}}, \\
& S(g=0)=\left\{\begin{array}{ll}
-N^{2} / 4 & (N \text { even }) \\
-\left(N^{2}-1\right) / 6 & (N \text { odd })
\end{array},\right.
\end{aligned}
$$

where Eq. (18) gives the large $N$ behavior. The parity dependence of Eq. (19) is caused by the negative eigenvalues of $E\left(g_{1}, g_{2}\right)$, giving rise to terms oscillating like $(-1)^{N}$.

Example 4. Take $A_{0}=\sigma_{+}, A_{1}=\sigma_{-}+\sqrt{g}(11+Z) / 2$. This MPS is the ground state of the following Hamiltonian:

$$
\begin{aligned}
H & =-\sum_{i} g\left(X_{i}+X_{i} Z_{i+1}+Z_{i} X_{i+1}+Z_{i} X_{i+1} Z_{i+2}\right) \\
& +\left(1+2 g^{2}\right) Z_{i}-2 Z_{i} Z_{i+1}-Z_{i} Z_{i+1} Z_{i+2} .
\end{aligned}
$$

The eigenvalues of $E\left(g_{1}, g_{2}\right)$ are $\left(0,0,\left(\sqrt{g_{1} g_{2}} \pm\right.\right.$ $\left.\sqrt{g_{1} g_{2}+4}\right) / 2$ ). Hence, the asymptotic behavior of $S(g)$ is again given by Eq. (18), while $S(0)=-N^{2} / 2$ for $N$ even and $S(0)=-\left(N^{2}-1\right) / 6$ for $N$ odd. Parity dependence arises from $\left(\sqrt{g_{1} g_{2}}-\sqrt{g_{1} g_{2}+4}\right) / 2=-1$ for $g_{1}=g_{2}=0$.

\section{B. Fidelity, concurrence, and single site entanglement}

While all the above examples fit in the same picture, which is typical of the second order quantum phase transitions already studied in Refs. 1,2, the next example, albeit trivial as matrix product state, features a different behavior, which will serve as a basis for some additional comments on the fidelity approach to QPTs.

Example 5. Take $D=d=2, A_{0}=\left(\begin{array}{cc}1 & 0 \\ 0 & 1+g\end{array}\right), A_{1}=$ $\left(\begin{array}{cc}g^{n} & 0 \\ 0 & 0\end{array}\right)$. The matrix $E\left(g_{1}, g_{2}\right)$ has the eigenvalues $1+g_{1}$, $1+g_{2},\left(1+g_{1}\right)\left(1+g_{2}\right)$, and $1+g_{1}^{n} g_{2}^{n}$. As discussed in Sec. III QPTs take place when two or more eigenvalues of $E_{\mathbb{1}}(g)=E(g, g)$ share the largest modulus. We then have to compare $1+g,(1+g)^{2}$, and $1+g^{2 n}$. Clearly, for $g<0$ one has $\lambda_{1}(g)=1+g^{2 n}$, while for $g \geq 0$ the dominant eigenvalue is determined by the ratio $r(g):=\left(1+g^{2 n}\right) /(1+g)^{2}$. The equation $r(g)=1$ can be rewritten as $g\left(g^{2 n-1}-g-2\right)=0$. Apart from the trivial solution $g_{c}=0$, for $n \geq 2$ a second solution $g_{c}^{\prime}>1$ exists, so that in the region $0 \leq g \leq g_{c}^{\prime}$ one has $\lambda_{1}(g)=(1+g)^{2}$, while for $g>g_{c}^{\prime}$ one obtains again $\lambda_{1}(g)=1+g^{2 n}$. For $n=1$ the point $g_{c}^{\prime}$ can be considered at infinity and the latter level crossing never occurs.

In order to evaluate the fidelity we have to substitute the above eigenvalues in Eq. (6). For simplicity, here we restrict our analysis to the fidelity of ground states belonging to the same phase, avoiding to discuss the effects shown in the inset of Fig. 1 for Example 1. In the phase corresponding to 


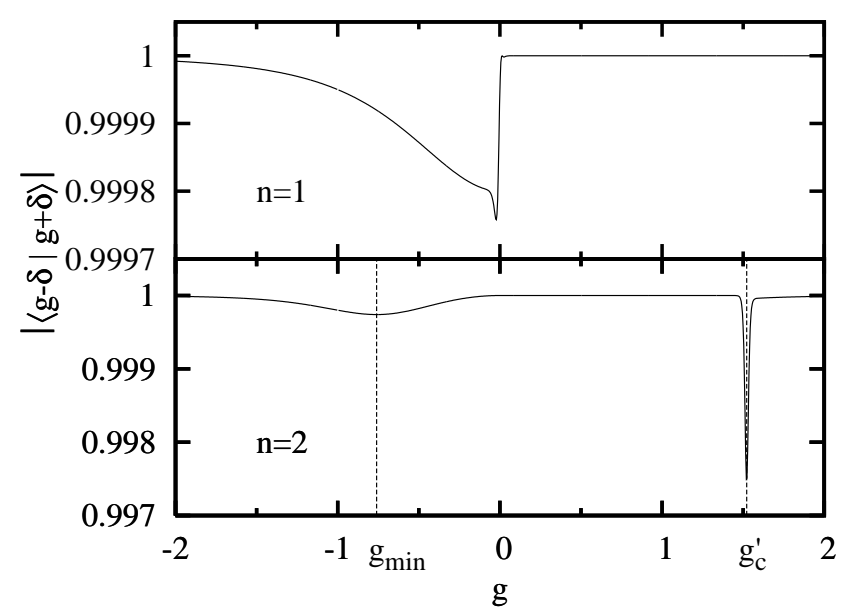

FIG. 3: Fidelity for the model given in Example 5, with $\delta=10^{-3}$ and $N=100 ; n=1$ (top) and $n=2$ (bottom).

the interval $g \in\left(0, g_{c}^{\prime}\right)$, where $\lambda_{1}(g)=(1+g)^{2}$, one has $\lambda_{1}\left(g_{1}, g_{2}\right)=(1+g-\delta)(1+g+\delta)$. The normalized fidelity in the TDL then results $\mathcal{F}(g ; \delta) \sim[(1+g-\delta)(1+$ $\left.g+\delta) / \sqrt{(1+g-\delta)^{2}(1+g+\delta)^{2}}\right]^{N}=1$, so that one has a constant phase in this region, due to the factorization of the largest eigenvalue of $E(g-\delta, g+\delta)$. Outside this region such a factorization is absent and the fidelity vanishes exponentially with the size of the system.

The asymptotic behavior of the second derivative $S(g)$ can be calculated accordingly. In the interval $g \in\left(0, g_{c}^{\prime}\right)$, where the eigenvalue $(1+g)^{2}$ dominates, one finds $S(g)=0$ in the TDL, while for $g \notin\left[0, g_{c}^{\prime}\right]$, where the leading eigenvalue is given by $1+g^{2 n}$, Eq. (10) yields the formula

$$
S(g) \sim-4 N n^{2} g^{2 n-2} /\left(1+g^{2 n}\right)^{2} .
$$

The latter function, which is evidently symmetric in $g$, has a minimum at $g_{\min }=-[(n-1) /(n+1)]^{1 / 2 n}$ (since $\left|g_{\min }\right|<$ $g_{c}^{\prime}$, the minimum position for $g>0$ is instead given by $g_{c}^{\prime}$ itself, as $S(g)$ increases monotonically for $g>g_{c}^{\prime}$ ). Note that, while $S(g)$ is continuous at $g=g_{c}=0$, where $S(0)=0$, at the second critical point $g=g_{c}^{\prime}$ one has the discontinuity $\lim _{g \rightarrow\left(g_{c}^{\prime}\right)^{-}} S(g)=0 \neq \lim _{g \rightarrow\left(g_{c}^{\prime}\right)}+S(g)=-4 N n^{2}\left(g_{c}^{\prime}+\right.$ 2) $/\left[g_{c}^{\prime}\left(1+g_{c}^{\prime}\right)^{4}\right]$, where the latter results is obtained by substituting the defining relation $r\left(g_{c}^{\prime}\right)=1$ into Eq. (21). Furthermore, exactly at the critical point $g_{c}^{\prime}$ one has the superextensive scaling $S\left(g_{c}^{\prime}\right) \sim-N^{2}\left[2 n-1+(n-1) g_{c}^{\prime}\right]^{2} /\left(1+g_{c}^{\prime}\right)^{4}$.

The finite size behavior of the fidelity is shown in Fig. 3 for $N=100$ and $\delta=10^{-3}$. Due to the approximated relation $\mathcal{F}(g ; \delta) \simeq 1+S(g) \delta^{2} / 2$ one can there recognize also the scaling of $S(g)$. In particular, in the lower panel where the case $n=2$ is plotted, the minimum at $g_{\min }$ and the superextensive scaling at $g_{c}^{\prime}(n=2) \simeq 1.521$ are evident.

For this simple example it is also possible to find a compact analytic expression for the ground state $|g\rangle$. Due to the commutativity $\left[A_{0}, A_{1}\right]=0$, one has $\sqrt{\mathcal{N}}|g\rangle=$ $\sum_{\boldsymbol{i} \in \mathbb{Z}_{2}^{N}} \operatorname{Tr}\left(A_{0}^{N-k} A_{1}^{k}\right)\left|i_{1} \ldots i_{N}\right\rangle$ with $k=\sum_{j=1}^{N} i_{j}$. Then, by using $\operatorname{Tr}\left(A_{0}^{N}\right)=1+(1+g)^{N}$ and $\operatorname{Tr}\left(A_{0}^{N-k} A_{1}^{k}\right)=g^{n k}$ for $k \neq 0$, one finds

$$
\begin{aligned}
\sqrt{\mathcal{N}}|g\rangle & =\left[1+(1+g)^{N}\right]|0\rangle^{\otimes N}+\sum_{k=1}^{N} g^{n k}\left(\begin{array}{c}
N \\
k
\end{array}\right)^{\frac{1}{2}}\left|D_{N}^{(k)}\right\rangle= \\
& =(1+g)^{N}|0\rangle^{\otimes N}+\left(1+g^{2 n}\right)^{N / 2}|\phi(g)\rangle^{\otimes N}, \quad \text { (22) }
\end{aligned}
$$

where $\left|D_{N}^{(k)}\right\rangle=\left(\begin{array}{c}N \\ k\end{array}\right)^{-\frac{1}{2}} \sum_{i \in I_{k}}\left|i_{1} \ldots i_{N}\right\rangle$ with $I_{k}=\{\boldsymbol{i} \in$ $\left.\mathbb{Z}_{2}^{N} \mid \sum_{j=1}^{N} i_{j}=k\right\}$ is a Dicke state ${ }^{10}$ and $|\phi(g)\rangle=(|0\rangle+$ $\left.g^{n}|1\rangle\right) / \sqrt{1+g^{2 n}}$ is a normalized single site state. Since the normalization is $\mathcal{N}=\operatorname{Tr} E_{\mathbb{1}}^{N}$, depending on the dominating eigenvalue of $E_{\mathbb{1}}$ three cases are possible in the TDL:

(i) $\lambda_{1}(g)=(1+g)^{2}>\lambda_{2}(g)$ and $|g\rangle \rightarrow|0\rangle^{\otimes N}$;

(ii) $\lambda_{1}(g)=1+g^{2 n}>\lambda_{2}(g)$ and $|g\rangle \rightarrow|\phi(g)\rangle^{\otimes N}$;

(iii) $\lambda_{1}(g)=\lambda_{2}(g)$ and, in the non-trivial case $g=g_{c}^{\prime}$, one has $^{14}|g\rangle \rightarrow\left(|0\rangle^{\otimes N}+|\phi\rangle^{\otimes N}\right) / \sqrt{2}$.

The above analysis can be summarized in the following table, where we recall the results for the largest transfer operator eigenvalue $\lambda_{1}(g)$ and for the large $N$ behavior of the function $S(g)$ and the ground state $|g\rangle$ :

\begin{tabular}{|c|c|c|c|}
\hline & $g \in\left[0, g_{c}^{\prime}\right)$ & $g=g_{c}^{\prime}$ & $g \notin\left[0, g_{c}^{\prime}\right]$ \\
\hline \hline$\lambda_{1}(g)$ & $(1+g)^{2}$ & $1+g^{2 n}=(1+g)^{2}$ & $1+g^{2 n}$ \\
\hline$S(g)$ & 0 & $\propto N^{2}$ & $\propto N$ \\
\hline$|g\rangle$ & $|0\rangle^{\otimes N}$ & $\left(|0\rangle^{\otimes N}+\left|\phi\left(g_{c}^{\prime}\right)\right\rangle^{\otimes N}\right) / \sqrt{2}$ & $|\phi(g)\rangle^{\otimes N}$ \\
\hline
\end{tabular}

It is worth noting that the apparent drop of the fidelity at $g_{\min }$ in the $g<0$ phase for $n \geq 2$ does not correspond to a QPT. Indeed, the scaling of $S\left(g_{\min }\right)$ does not present any peculiar behavior with respect to the rest of the $g<0$ phase. On the contrary, the scaling of $S$ changes its nature at $g=0$ and $g=g_{c}^{\prime}$, highlighting the transitions, although in a very different way from the typical behavior observed in the previous second order QPTs. The transition at $g=0$ corresponds to a discontinuity in $\partial_{g}^{n}\langle X\rangle$ (Ref. 4), while that at $g=g_{c}^{\prime}$ to a discontinuity of $\langle X\rangle$ itself $^{15}$. Due to the permutation symmetry arising from the commutativity of $A_{0}, A_{1}$, the correlation length cannot here be defined (the usual formula $\xi_{c}=1 / \ln \left|\lambda_{1} / \lambda_{2}\right|$ does not hold). In practice, the transitions of Example 5 appear to be of first order.

For this example we also investigate concurrence and single site entanglement, mainly focusing on the critical point $g_{c}=0$. Of course, the fact that the state is factorized in the TDL trivially implies the vanishing of these quantities in this limit. We are however interested in the finite size scaling of the derivatives of these measures, in the spirit of Ref. 6 . We are motivated by various aspects. First, as mentioned before, in MPS-QPTs the block entanglement entropy does not exhibit the logarithmic divergence observed in other quantum phase transitions, posing the question whether other entanglement properties usually signaling quantum criticality behave differently in this case. Second, we are interested in comparing the effectiveness of the fidelity approach against other quantum information-theoretical methods. The simple nature of this example allows for a fully analytical treatment.

Concurrence. We compute the concurrence for the reduced density matrix of 2 qubits in the chain (the choice of 
the qubits is unimportant, due to the permutation symmetry mentioned above). The density matrix of two spins $\rho^{(2)}$ is obtained by tracing the initial $\rho=|g\rangle\langle g|$ over all the other spins in the chain. By exploiting the symmetries arising from the commutation relations $\left[A_{i}, A_{j}\right]=0$ and recalling that $\rho^{(2)}=\sum_{i, j, k, l=0}^{1} \rho_{i j, k l}^{(2)}|i j\rangle\langle k l|$ is a $4 \times 4$ real symmetric matrix, one finds $\rho_{i j, k l}^{(2)}=\rho_{j i, k l}^{(2)}=\rho_{i j, l k}^{(2)}$. Together with the normalization $\operatorname{Tr} \rho^{(2)}=1$ this implies that only 5 entries of $\rho^{(2)}$ are independent, e.g., $\rho_{00,00}^{(2)}, \rho_{00,01}^{(2)}, \rho_{00,11}^{(2)}, \rho_{01,01}^{(2)}, \rho_{01,11}^{(2)}$, significantly simplifying the calculation in the standard basis. Otherwise, one can use Eq. (22), easily obtaining

$$
\begin{aligned}
\rho^{(2)}= & {\left[(1+g)^{2 N}|00\rangle\left\langle 00\left|+\left(1+g^{2 n}\right)^{N}\right| \phi \phi\right\rangle\langle\phi \phi|\right.} \\
& \left.+(1+g)^{N}\left(1+g^{2 n}\right)(|00\rangle\langle\phi \phi|+| \phi \phi\rangle\langle 00|)\right] / \mathcal{N}(g) .
\end{aligned}
$$

Having $\rho^{(2)}$, the concurrence for two qubits is defined as 9 $C=\max \left\{0, \sqrt{\mu_{1}}-\sqrt{\mu_{2}}-\sqrt{\mu_{3}}-\sqrt{\mu_{4}}\right\}$, where $\mu_{i}$ 's are the eigenvalues, in decreasing order, of the operator $\rho^{(2)}\left(\sigma_{y} \otimes\right.$ $\left.\sigma_{y}\right)\left(\rho^{(2)}\right)^{*}\left(\sigma_{y} \otimes \sigma_{y}\right)$. We finally have:

$$
C(g)=\frac{2 g^{2 n}\left|(1+g)^{N}\right|}{\mathcal{N}(g)} .
$$

In the thermodynamic limit both $C(g)$ and its first derivative vanish, so that the kind of transition signatures found, e.g., in Ref. 6 are absent here. However, for $n=1$, the fourth derivative $\left.\partial_{g}^{4} C\right|_{g=0}$ has a divergence in the TDL; for $n \geq 2$ the singularity is present in even higher derivatives.

Single site entanglement. This quantity is the von Neumann entropy of a single spin $\mathcal{S}=-\operatorname{Tr}\left(\rho^{(1)} \log _{2} \rho^{(1)}\right)$, where $\rho^{(1)}$ is the reduced density matrix of a single site. Again from Eq. (22) one finds

$$
\begin{aligned}
\rho^{(1)}= & {\left[(1+g)^{2 N}|0\rangle\left\langle 0\left|+\left(1+g^{2 n}\right)^{N}\right| \phi\right\rangle\langle\phi|\right.} \\
& \left.+(1+g)^{N} \sqrt{1+g^{2 n}}(|0\rangle\langle\phi|+| \phi\rangle\langle 0|)\right] / \mathcal{N}(g),
\end{aligned}
$$

whose eigenvalues are $\lambda_{ \pm}=\left(1 \pm \sqrt{1-4 \operatorname{det} \rho^{(1)}}\right) / 2$, with $\lambda_{+}=1-\lambda_{-}$and

$$
\operatorname{det} \rho^{(1)}=\frac{g^{2 n}(1+g)^{2 N}\left[\left(1+g^{2 n}\right)^{N-1}-1\right]}{\mathcal{N}^{2}(g)} .
$$

The single site entanglement entropy $\mathcal{S}=-\lambda_{+} \log _{2} \lambda_{+}-$ $\lambda_{-} \log _{2} \lambda_{-}$vanishes in the thermodynamic limit at $g=0$, together with its first derivative. For $n=1$, one finds a divergence in $\left.\partial_{g}^{4} \mathcal{S}\right|_{g=0}$, while the divergence is shifted to higher derivatives for $n \geq 2$, similarly to the concurrence. The considered divergence is however due to the functional form of the von Neumann entropy. Indeed, $\lambda_{-} \rightarrow 0$ for $g \rightarrow 0$, giving rise to a singularity in the logarithm.
We conclude this analysis by briefly discussing the TDL of these entanglement measures at the critical point $g=$ $g_{c}^{\prime}$, where the state is not factorized (see the table above). The single site reduced density matrix in the TDL is simply $\rho^{(1)}\left(g_{c}^{\prime}\right)=(|0\rangle\langle 0|+| \phi\rangle\langle\phi|) / 2$ and $\operatorname{det} \rho^{(1)}\left(g_{c}^{\prime}\right)=[1-1 /(1+$ $\left.\left.g_{c}^{\prime}\right)^{2}\right] / 4$. Then $\lambda_{ \pm}\left(g_{c}^{\prime}\right)=\left[1 \pm 1 /\left(1+g_{c}^{\prime}\right)\right] / 2$ and $\mathcal{S}\left(g_{c}^{\prime}\right) \neq 0$. Note however that $C\left(g_{c}^{\prime}\right)=0$ in the TDL, reminiscent of the behavior of the GHZ state. This can be seen from the simple TDL of Eq. (23), namely, $\rho^{(2)}\left(g_{c}^{\prime}\right)=(|00\rangle\langle 00|+| \phi \phi\rangle\langle\phi \phi|) / 2$.

\section{CONCLUSIONS}

In conclusion we have investigated the quantum fidelity of slightly different matrix product states dependent on a parameter $g$ in the context of quantum phase transitions. For generic MPSs, the overlap can be related analytically to the eigenvalues of a generalized transfer operator, among which the largest in modulus plays a crucial role. If the latter is non-degenerate (in modulus), the fidelity typically exhibits an exponential decay in the thermodynamic limit and its second derivative is proportional to the size $N$ of the system. If instead more eigenvalues share the largest modulus, a quantum phase transition can take place and the fidelity second derivative $S(g)$ generally scales with $N^{2}$. We have demonstrated this behavior in the exhaustive analysis of some simple examples, taken from Ref. 4, where possible exceptions have also been pointed out. Moreover, we have shown that the second derivative of the fidelity is a useful quantity for the quantitative analysis of the scaling properties of the system at the transition point. From the finite size scaling behavior of $S(g)$ we have indeed estimated the critical exponent for the correlation length, finding agreement with the explicit calculation. Finally, for one of the considered examples, we have analyzed both concurrence and single site entanglement. Although the latter quantities did provide signatures of the undergoing quantum phase transitions, this information was hidden in high order derivatives, making it much more difficult to extract than the fidelity (or its second derivative).

The fidelity analysis has the advantage of providing a unified framework to detect very different types of phase transitions. For the concrete examples analyzed here, singularities in the fidelity are related to level crossings in the transfer operator, recovering the known transition mechanism for matrix product states. The results presented here further contribute to demonstrate the generality of the fidelity approach to quantum phase transitions $\stackrel{11}{11}$, supporting the findings of Refs. 1. 2 .

\section{Acknowledgments}

We thank L. Amico and P. Giorda for valuable discussions and comments.
1 P. Zanardi and N. Paunković, Phys. Rev. E 74, 031123 (2006); P. Zanardi, M. Cozzini, and P. Giorda, quant-ph/0606130

2 M. Cozzini, P. Giorda, and P. Zanardi, quant-ph/0608059
3 I. Affleck, T. Kennedy, E.H. Lieb and H. Tasaki, Phys. Rev. Lett. 59, 799 (1987); Commun. Math. Phys. 115, 477 (1988); M. Fannes, B. Nachtergaele and R.F. Werner, Europhys. Lett. 10, 
633 (1989); Commun. Math. Phys. 144, 443 (1992); S. Östlund and S. Rommer, Phys. Rev. Lett. 75, 3537 (1995); S. Rommer and S. Östlund, Phys. Rev. B 55, 2164 (1997); D. Pérez-García, F. Verstraete, M.M. Wolf, and J.I. Cirac, quant-ph/0608197.

${ }^{4}$ M.M. Wolf, G. Ortiz, F. Verstraete, J.I. Cirac, Phys. Rev. Lett. 97, 110403 (2006).

5 G. Vidal, J.I. Latorre, E. Rico, and A. Kitaev, Phys. Rev. Lett. 90, 227902 (2003); G. Refael and J.E. Moore, Phys. Rev. Lett. 93, 260602 (2004); V. Korepin, Phys. Rev. Lett. 92, 096402 (2004).

${ }^{6}$ A. Osterloh, L. Amico, G. Falci, and R. Fazio, Nature 416, 608 (2002).

7 H.-Q. Zhou and J.P. Barjaktarevic, private communication.

${ }^{8}$ M.E. Fisher and M.N. Barber, Phys. Rev. Lett. 28, 1516 (1972); M.N. Barber, in Phase Transitions and Critical Phenomena, edited by C. Domb and J.L. Lebowitz (Academic Press, London, 1983), Vol. 8, p. 145.

9 W.K. Wootters, Phys. Rev. Lett. 80, 2245 (1998).

${ }^{10}$ R.H. Dicke, Phys. Rev. 93, 99 (1954).
11 By using Uhlmann's fidelity for mixed states, one can extend this kind of analysis to finite temperature systems and therefore even to classical phase transitions. See P. Giorda and P. Zanardi, in preparation.

12 Considering the fidelity $|\langle g-\delta \mid g+\delta\rangle|$ corresponds to taking a variation $2 \delta$ between the states. This gives rise to the factor 4 in formula (8) and differs from Refs. 1,2.

13 The correlation length in MPSs is usually given by the formula $\xi_{c}=1 / \ln \left|\lambda_{1} / \lambda_{2}\right|$, where $\lambda_{1}\left(\lambda_{2}\right)$ is the eigenvalue of $E_{\mathbb{1}}(g)$ of (second) largest modulus. In this case, however, the degeneracy of $\lambda_{2}=\lambda_{3}=-1$ does not allow to apply this expression.

14 Note that $|0\rangle^{\otimes N}$ and $\left|\phi\left(g_{c}^{\prime}\right)\right\rangle^{\otimes N}$ become orthogonal in the TDL, in accordance with the normalization factor $1 / \sqrt{2}$.

15 In the limit $N \rightarrow \infty$ one has $\langle X(g)\rangle \rightarrow 0$ for $g \in\left[0, g_{c}^{\prime}\right)$, $\left\langle X\left(g_{c}^{\prime}\right)\right\rangle \rightarrow\left(g_{c}^{\prime}\right)^{n} /\left[1+\left(g_{c}^{\prime}\right)^{2 n}\right]$, and $\langle X(g)\rangle \rightarrow 2 g^{n} /\left(1+g^{2 n}\right)$ for $g \notin\left[0, g_{c}^{\prime}\right]$. Hence, for example, $\lim _{g \rightarrow 0^{-}} \partial_{g}^{n}\langle X\rangle=2 n$ !, while $\lim _{g \rightarrow 0^{+}} \partial_{g}^{n}\langle X\rangle=0$. 